\title{
El desarrollo y legitimación de las organizaciones del tercer sector en la Argentina. Hacia la definición de un isomorfismo periférico ${ }^{1}$
}

\section{Introducción}

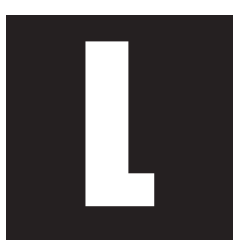

as organizaciones del tercer sector en la Argentina no son un fenómeno nuevo, sino que existen hace mucho aunque adopten diferentes nombres: fundación, sociedad de fomento, sociedad de beneficencia, organizaciones de la sociedad civil, etc. Pero es recién a partir de la década del noventa que esas organizaciones empiezan a ser estudiadas por la comunidad académica, que ven en ellas un actor fundamental para el cambio social.

Durante el período colonial y hasta avanzado el siglo XIX encontramos instituciones fundamentalmente de tipo eclesiástico (talescomo hermandades y cofradías) y posteriormente también organizaciones tales como la Sociedad de Beneficencia. El arribo de las oleadas de inmigración masiva da lugar al surgimiento de una cantidad de asociaciones de socorros mutuos de base étnica que en algunos casos evolucionarán hasta convertirse en importantes instituciones mutuales y hospitalarias. Las bibliotecas populares y los clubes

* PhD pela Notre Dame U niversity, Estados U nidos; diretor do IDICSO (Instituto de Investigación en Ciencias Sociales) da Universidad del Salvador, Buenos Aires; professor titular de Teoria Social e Política Social nessa mesma universidade. Argentina ** Bacharel em Letras pela Universidade Ibero - Americana de São Paulo. Atualmente elaborando tese de graduação para concluir o curso de Sociologia da Universidad del Salvador, Buenos Aires; aluna especial do curso do Dr. Paulo M eneses na Universidade de São Paulo; assistente de pesquisa do IDICSO (Instituto de Investigación en Ciencias Sociales), da Universidad del Salvador.

1 Agradecemos los comentarios a versiones anteriores de este articulo a Marcelo Salas y Ricardo Murtagh. 
Sociologias, Porto Alegre, ano 8, no 16, jul/dez 2006, p. 216-249

deportivos son también una novedad de las primeras décadas del siglo XX. El Estado conservador de entonces es básicamente ajeno a estos desarrollos que incluso reprime en algunos casos por sus relaciones con el incipiente movimiento obrero.

El advenimiento del peronismo señala un período distinto para la participación y las organizaciones sociales. La satisfacción de muchas demandas sociales postergadas pasa a ser unificada por el Estado a través de instituciones tales como la Fundación Eva Perón. El intento por constituir un Estado del Bienestar impulsa el péndulo de lo social hacia la esfera gubernamental, si bien puede señalarse como una excepción la expansión de las actividades sociales de muchas organizaciones sindicales.

Con posterioridad al golpe de 1955 se establece Caritas como la agencia de la Iglesia Católica a cargo de las cuestiones sociales. Como mencionamos anteriormente, el rol de las instituciones religiosas en actividades caritativas ha sido y es muy importante en Argentina. A nivel mundial, Caritas Internationalis fue creada en los años 50, con el objetivo de "ayudar sus miembros a irradiar caridad y justicia social en le mundo". Caritas Argentina fue creada inicialmente con el nombre de "Fraterna ayuda Cristiana", cambiando su nombre al que hoy conocemos 11 años después. Como parte de una organización tan grande como es la Iglesia Católica Romana, Cáritas actúa de modo bastante heterogéneo en muy diferentes niveles (nacional, regional, diocesano y parroquial) y la mayor parte de sus recursos viene de donaciones que son divididos en tres partes iguales entre los niveles parroquial, diocesano y nacional.

La última dictadura militar (1976-83) llevó al extremo la represión a las organizaciones políticas y sociales en todo el país. Corresponde a este difícil período el surgimiento de las internacionalmente reconocidas organizaciones dedicadas a la defensa de los derechos humanos. Luego del retorno a la democracia, se dio una intensa creación de organizaciones no 
Sociologias, Porto Alegre, ano 8, no 16, jul/dez 2006, p. 216-249

gubernamentales vinculadas a cuestiones tales como la defensa del consumidor, el medio-ambiente, el género, etc. Sin embargo, son las organizaciones dedicadas a problemáticas derivadas de la creciente pobreza y exclusión social las que fueron cobrando mayor relevancia y visibilidad.

Es importante aclarar que en los tres periodos mencionados, las organizaciones del tercer sector siempre se encargaron de suplir deficiencias dejadas por el Estado, tanto en aspectos educativos, culturales y recreativos, como proveedores de asistencia básica en el área de la salud, vivienda y alimentación. Aún así, el Estado siempre ha sido el principal proveedor de fondos para las actividades de dichas organizaciones, aunque en la década del noventa, esencialmente, se produce un cambio radical en relación a períodos anteriores. Nuevos actores entran al campo del tercer sector, como organismos multilaterales de crédito, por ejemplo, el Banco Mundial, el Banco Interamericano de Desarrollo, etc. Por otra parte, las organizaciones empiezan a desarrollar actividades donde venden sus servicios para conseguir fondos: es más, la recaudación de fondos o el fund raising comienza a ser un elemento clave de las organizaciones del tercer sector. Al mismo tiempo, éstas empiezan a funcionar como organizaciones consultoras, tanto en lo que se refiere a la necesidad de conocimiento acerca del campo como a las tecnologías más adecuadas para implementar políticas sociales (ya que esas organizaciones, principalmente las de base, tienen un contacto muy cercano con sus "beneficiarios").

Hablar de ONGs en la Argentina es referirse a un fenómeno muy dispar, a muchas formas de organizaciones a la vez. Sin embargo, para acotar nuestro objeto, vamosa referirnos acá principalmente a "asociaciones sin fines de lucro legalmente constituidas que brindan servicios, movilizan intereses, alientan la autonomía y actúan como propulsoras para mejorar las condiciones de vida y oportunidad de los ciudadanos" (Reilly, 1995). En realidad, dada la diversidad de términos en uso que aluden a organizaciones 
en extremo heterogeneas utilizamos aquí el término tercer sector pues, a pesar de las diferencias que caracterizan a estas instituciones en diferentes países, comparten rasgos comunes que hacen posible considerarlas parte de un "sector" social distinguible: se trata de organizaciones que operan por fuera del aparato estatal, que no distribuyen beneficios y a los que los ciudadanos son libres de asociarse 0 no en la persecución de objetivos comunes. (Salomon \& Anheiner, 1996).

El objetivo central de este estudio fue analizar cómo se dio el proceso de institucionalización de las organizaciones del tercer sector en la Argentina en la década del 90 , basándonos en la bibliografía producida acerca de y por dichas organizaciones. Para ello, en una primera etapa, se hizo un relevamiento de toda la bibliografía disponible acerca de organizaciones del tercer sector, publicada a fines de los 80 y, principalmente, en los 90, en diez bibliotecas universitarias de la ciudad de Buenos Aires y Gran Buenos Aires. Esta etapa culminó con una publicación online de la bibliografía estudiada. ${ }^{2}$ Luego, en una segunda etapa, estudiamos el material encontrado en función de la teoría neoinstitucional, que nos proporcionaba herramientas muy útiles para analizar el proceso de institucionalización de las organizaciones en cuestión, principalmente en lo que se refería a la adecuación de las categorías a los datos. Después, lo que se buscó fue adaptar la teoría a los datos que, a su vez, generaron nuevas dudas y planteos respecto al proceso en cuestión. ${ }^{3}$

$\mathrm{H}$ ay dos aspectos importantes, que fueron planteados como retos para llevar a cabo este estudio: la heterogeneidad de los datos y de las organizaciones a las que nos referimos. Por un lado, el hecho de que nos

2 La bibliografía completa puede ser consultada en la página web del IDICSO : http://www.salvador.edu.ar/csoc/idicso/docs/ aongpp001.pdf

3 Este estudio se hizo en el marco del Proyecto Binacional Brasil-Argentina: "Redes organizacionalesy patrones institucionales de O NGs: un estudio comparativo entre la Argentina y Brasil" que contó con el apoyo de la Fundación Antorchas. Participaron los siguientes estudiantes de la Facultad de Ciencias Sociales de la Universidad del Salvador: Luciana Castronuovo, M arcelo Salas, Gabriella Panelli, Martín Gaspar Arslanian y M aria Laura Videla. 
Sociologias, Porto Alegre, ano 8, no 16, jul/dez 2006, p. 216-249

manejábamos con datos escritos acerca de o por las organizaciones del tercer sector determinaba, hasta cierto punto, la heterogeneidad de nuestros datos, ya que, fundamentalmente, esa bibliografía oscilaba entre textos de organismos internacionales acerca de las organizaciones del tercer sector, manuales prescriptivos, artículos enalteciendo el papel de dichas organizaciones, investigaciones académicas, entre otros. Por otro lado, la heterogeneidad de las mismas organizaciones se hace más patente aún ya que en los en los textos tampoco hay referencias explícitas acerca de qué tipo de organizaciones abarcan: si O N Gs de promoción y desarrollo, cooperativas, asociaciones, etc., sino que a menudo se remiten a todo tipo de organización que no sea de mercado.

\section{El enfoque neo-institucional}

El estudio de las instituciones ha sido llevado a cabo por diversas áreas científicas como la economía, la psicología, las ciencias políticas y el derecho. Nos centraremos en el enfoque institucional desarrollado por teóricos de la sociología. Los primeros teóricos del análisis institucional en sociología hacían hincapié en el hecho de que el comportamiento de los individuos estaba determinado por las instituciones al mismo tiempo que éstas se anclaban en el comportamiento estandarizado de los individuos. Sin embargo, diferentes autores ponían énfasis en diversos aspectos, estructurales o simbólicos, que consideraban claves para el proceso de institucionalización: los sistemas simbólicos (D urkheim), el significado puesto por los actores en la acción social y la racionalidad (Weber), la autoridad moral (Parsons), la interdependencia entre el individuo y la sociedad (G.H. M ead), la realidad como una construcción social (Berger y Luckmann), entre otros. Es decir, alternaban entre un enfoque que ponía el actor como creador de la estructura o ésta como determinante de la acción de aquél. 
Hoy, dejando de lado un énfasis u otro, lo que se observa es que hay un intento de fusionar ambos aspectos, estructurales y constructivistas, para explicar la realidad social y la institución como un proceso fundamental de ella, como se puede ver en autores como Giddens (1984) en su teoría de la estructuración y Bourdieu (1980) en sus análisis acerca del proceso de institucionalización como realidad objetivada e incorporada. Este último autor explica: "sólo se puede, pues, comprender el funcionamiento de lasinstituciones burocráticas si se va más allá de la oposición ficticia entre una visión 'estructuralista', por un lado (...) y, por otro lado, una visión 'interaccionista' (...), ignorando tanto las condiciones sociales de producción de los agentes ... como las condiciones institucionales del ejercicio de su función..." .

El estudio de las instituciones puede estar basado en tres aspectos principales (Scott, 1995), considerados esenciales para que un sistema 0 comportamiento dados se institucionalicen, y que se refieren a cuáles elementos entran en juego en dicho proceso: regulativo, normativo y cognitivo. El enfoque regulativo se basa en el hecho de que lasinstituciones conforman el comportamiento del individuo, y que reglas expresas o implícitas (sanciones legales o culturales, por ejemplo) son mecanismos claves a la hora de estandarizar e institucionalizar un patrón de comportamiento. Desde este enfoque, los individuos actúan según un cálculo de costos y beneficios y siguen las reglas llevados por sus propios intereses. En el enfoque normativo el énfasis está puesto tanto en los valores como en las normas que definen la mejor manera de hacer las cosas. Estas normas y valores (que pueden 0 no ser impuestos desde afuera) condicionan la manera en que los individuos van a actuar, pero al mismo tiempo les favorece al proveer un marco donde la acción social pueda desarrollarse libremente, pero siempre adentro del marco normativo en cuestión.

A su vez, el abordaje cognitivo, característico del enfoque neoinstitucional, enfatiza tanto aspectos simbólicos como estructurales para la 
Sociologias, Porto Alegre, ano 8, no 16, jul/dez 2006, p. 216-249

conformación y mantenimiento de las instituciones. La concepción de Berger y Luckmann (1967) atraviesa la mayoría de los estudios realizados en campo del análisis neoinstitucional, y preconiza que las instituciones se forman a través de sistemas compartidos de definiciones creados intersubjetivamente, pero que luego se tipifican, se objetivizan y se externalizan. Así, lo que se construye intersubjetivamente parece ser dado objetivamente. Asimismo, las características de lo construido adquieren una configuración específica según varían el tiempo y el espacio.

Para estos autores, la legitimación es una objetivación de significados compartidos que pasan a estar disponibles de manera plausible al conjunto de los individuos, otorgando, de esta forma, cierta justificación al orden institucional en cuestión. Por lo tanto, una vez que las organizaciones se hayan legitimado, logran imponerse frente a las demás organizaciones 0 actores en general del campo como organizaciones válidas.

Autores como Meyer y Rowan así como Di Maggio y Powell hacen hincapié en los sistemas culturales que les son impuestos o que son adoptados por el individuo o por organizaciones. Consideran que el rasgo distintivo de cualquier institucionalización es el isomorfismo, que es un proceso de homogeneización que puede originarse de dos modos: de la competición y adecuación de organizaciones individuales a cambios en el mercado, lo que se denomina isomorfismo competitivo; o de la competición por diferentes factores como influencia política, búsqueda de legitimación, etc., llamado isomorfismo institucional, que es el que usaremos para abordar el caso de las organizaciones del tercer sector (Powell y Di M aggio, 1991; Scott, 1995). Este segundo tipo de isomorfismo puede darse de diferentes maneras: o las organizaciones más grandes o poderosas imponen sus propias pautas a la sociedad en general (por ejemplo, cuando exigen que susfuncionariostengan determinadas credenciales- M.B.A., doctorados, etc.- para ejercer ciertos puestos); también puede darse porque las organizaciones más grandes y 
poderosas les impongan sus propias pautas a sus proveedores, por ejemplo, que se adapten a sus estructuras (Meyer y Rowan, 1991). De cualquier modo, siempre se trata de una respuesta de la estructura organizacional al ambiente institucional.

Sin embargo, las organizaciones no se constituyen y se desarrollan aisladas de su entorno, sino que interactúan con él de forma bastante intensa y las características de esa interacción van a tener diversos efectos en cómo se estructuran los elementos de dichas organizaciones.

A medida que un campo organizacional se va conformando, las nuevas organizaciones a menudo toman como ejemplo a otras más antiguas, ya exitosas. Los elementos que son incorporados pueden referirse a las normas jurídicas existentes, a patrones organizacionales, a la imagen institucional, etc. Así, las nuevas organizaciones tienden a adoptar mitos y ceremonias (M eyer y Rowan, 1991) previamente racionalizados e institucionalizados que van a proporcionarles legitimidad. Frente a esta necesidad, la eficiencia resulta menos relevante; más bien se mantienen mecanismos que garanticen la legitimidad, a través de dichos mitos y ceremonias que son compartidos, de modo que las organizaciones se hacen cada vez más homogéneas. Esos mitos son considerados, consciente 0 inconscientemente, racionales y efectivos. Por otro lado, el costo puede ser alto para aquellas organizaciones en vías de institucionalización que no adopten dichos mitos en la búsqueda por la legitimación.

Por lo tanto, las organizaciones en un incipiente proceso de institucionalización, en su búsqueda por la legitimidad, sufren procesos isomórficos que les proveen dos ventajas principales: ser reconocidas como legítimas o válidas por su entorno, lo que les permite, entre otras cosas, la expansión o el éxito y, además, disminuir la incertidumbre. La incertidumbre puede estar dada por factores tanto internos como externosa la organización. En el primer caso, al que Scott se refiere como entorno organizacional, 
Sociologias, Porto Alegre, ano 8, no 16, jul/dez 2006, p. 216-249

pueden mencionarse las dificultades de solvencia, luchas internas por el poder o el uso de nuevas tecnologías. En el caso de incertidumbres relacionadas al entorno institucional, está la búsqueda de legitimación, la relación con el mercado o clientes, la influencia de decisiones políticas y, para organizaciones del tercer sector más específicamente, la necesidad de obtener financiación.

A medida que incorporan mitos institucionales, las organizaciones tenderían a dejar la eficiencia en un segundo plano, llevando a ciertas inconsistencias donde, básicamente, las reglas institucionaleso ceremoniales pueden entrar en conflicto con el quehacer cotidiano de las organizaciones. En caso de que se opte por privilegiar la eficiencia, a su vez, la organización podría estar arriesgando su legitimidad.

Como se mencionó antes, el análisis institucional puede enfatizar diferentes elementos según la apuesta en cuáles son los aspectos predominantes en un proceso de institucionalización. En base a ello, Powell y DiM aggio señalan que el proceso de isomorfismo puede presentar tres diferentes mecanismos: coercitivo, mimético y normativo. El isomorfismo coercitivo "resulta de presiones tanto formales como informales que se expresan como una fuerza, persuasión o invitación a coludirse". Ello puede darse debido a un dictamen legal (por ejemplo, si los dispositivos de seguridad de los autos son requeridos por ley), a imposiciones de la casa matriz so bre sus sucursales y proveedores; la necesidad de adoptar cierta jerarquía para conseguir recursos económicos, etc.. Scott (1995) hace una diferenciación entre "imposición por medio de la autoridad" e "imposición por medio del poder coercitivo", donde la primera se encontraría con menores resistencias y con un mayor nivel de cumplimiento que la segunda.

A su vez, el isomorfismo mimético es una respuesta a la incertidumbre; las organizaciones copian de otras más exitosas modelos de gerenciamiento, usos de tecnologías e innovaciones, lo que les puede favorecer, además de la legitimación, el ahorro en término de costos. 
Finalmente, el isomorfismo normativo, según los autores, alude a la profesionalización, es decir, a medida que las organizaciones contratan gerentes y personal jerárquico de la misma rama de formación académica, esos profesionales ya pasan por una homogeneización de antemano, pues comparten un mismo lenguaje, mismas maneras de encarar y solucionar problemas, etc..

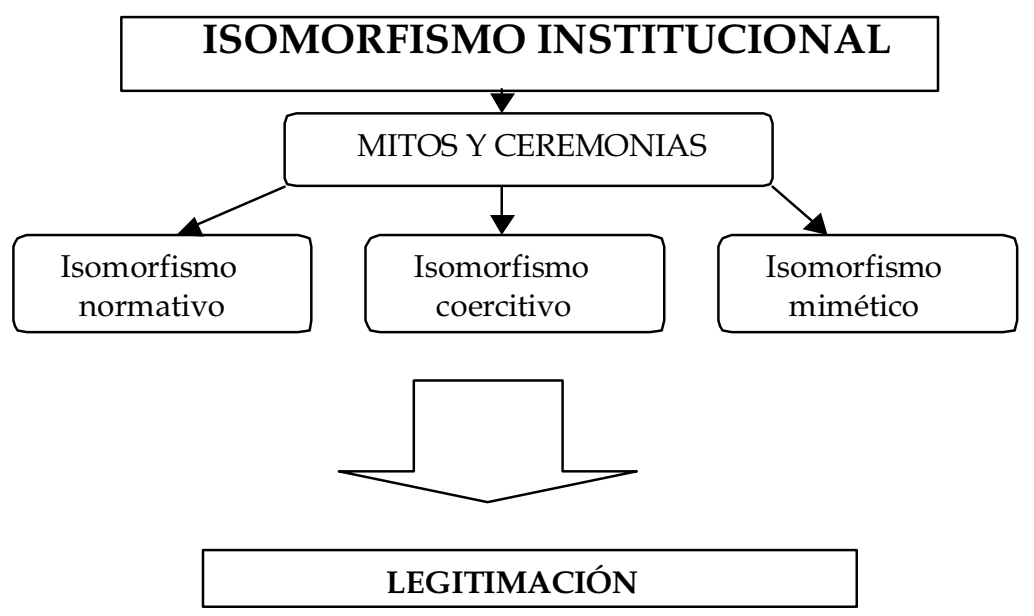

El enfoque neoinstitucional nos parece especialmente interesante para observar el caso de las organizaciones del tercer sector en la Argentina, dado que esun campo organizacional aún en formación y donde la estructura de relaciones que se da tanto al interior como extra campo son definitorias para la conformación del mismo. Ello, a su vez, va a determinar los procesos a través de los cuales dichas organizaciones adquieren creciente legitimidad. Además, el hincapié que este enfoque hace en los aspectos culturales y simbólicos de la institucionalización nos permite analizar a las organizaciones 
Sociologias, Porto Alegre, ano 8, no 16, jul/dez 2006, p. 216-249

del tercer sector desde sus rasgos locales; es decir, si bien las categorías de análisis propuestas por los teóricos neoinstitucionales sin duda son un gran aporte, las organizaciones y, más específicamente, las organizaciones del tercer sector en la Argentina adquieren características propias debido a las definiciones compartidas acerca de qué es o debería ser una organización legítima.

\section{El Entorno de las organizaciones del tercer sector en la} Argentina

El entorno con el cual las organizaciones del tercer sector interactúan en Argentina es dista batante de aquél de las organizaciones privadas, por un lado, y más aún del entorno organizacional de la sociedad estadounidense donde los autores antedichos desarrollaron sus teorías. Para dar cuenta del proceso isomórfico por el cual las organizaciones del tercer sector argentinas pasan, es menester detallar las especificidades de su entorno, ya que, vale recordar, la relación con el entorno es un aspecto crucial de la teoría neoinstitucional.

Primeramente, el mismo desarrollo del tercer sector y sus organizaciones, está vinculado a distintos cambios ocurridos en la sociedad Argentina a partir de 1976 y principalmente durante los años 90, cuando el Estado de bienestar entra en crisis y da lugar a un "modelo neoliberal excluyente". En él, según los preceptos del Consenso de Washington, se lleva a cabo una reforma del Estado basada en la liberalización financiera, la reestructuración del aparato del Estado basada en la eficacia y en la eficiencia, y la búsqueda de mayor competitividad en el libre mercado. Asimismo, un cambio importante fue la descentralización, que se llevó a cabo con el auspicio de los organismos multilaterales, donde actividades que eran anteriormente realizadas por el gobierno fueron transferidas a las manos de la 
sociedad civil y, más que nada, de las organizaciones del tercer sector como su representante. La dificultad principal está en que esto se dio sin una previa preparación del nuevo sector encargado de diversas tareas, y sin tener en cuenta su capacidad para responder a las nuevas demandas. A estas organizaciones les incumbe, entonces, implementar o monitorear muchas de las políticas sociales, de manera focalizada y descentralizada. Es más, en la década del 90 hubo un importante incremento de esas organizaciones que vinieron, por un lado, a suplir una demanda por parte del Estado que empezó a tercerizar algunos de sus servicios y programas y también, por el otro, a paliar los efectos de la crisis y actuar allí donde el Estado era ineficiente o inexistente. Sin embargo, hay dos problemas claves que esta nueva situación genera, como señala Murtagh(2002), que es la necesidad que el sector tiene de recursos externos y su independencia.

La dificultad para obtener financiación efectivamente resultó ser un problema: las organizaciones "sufren una tensión entre sentirse parte de una estrategia de desmembramiento del Estado (...) y el riesgo de mercantilizar sus actividades e insertarse en el mercado (...) como una forma de sobrevivir económicamente y mantener niveles de autonomía" (Arroyo, sin año). Así, las organizaciones tuvieron que rebuscarlas para conseguir financiación para sus proyectos, llevando a cabo diversas actividades de venta de servicios, consultorías, etc., concentradas bajo el la idea de fund raising. Esta es un área encargada de buscar financiación, la que comúnmente recurre a los organismos multilaterales de crédito, como el Banco Interamericano de Desarrollo y el Banco Mundial, y agencias internacionales en general, pero el Estado sigue siendo el actor principal a la que las organizaciones del tercer sector recurren no sólo para solicitar financiación sino también para otros tipos de ayuda, como alimentos, locales para diferentes actividades (centro de jubilados, bibliotecas populares, centros de rehabilitación, etc). No obstante, la inestabilidad de los recursos es una característica de las organizaciones del tercer sector argentinas. 
Sociologias, Porto Alegre, ano 8, no 16, jul/dez 2006, p. 216-249

Es importante mencionar que el mismo Estado empieza a ser cada vez más dependiente de los organismos multilaterales de crédito, principalmente del Fondo Monetario Internacional y del Banco Mundial, que van imponiendo reglas para las políticas públicas y también para el formato del aparato estatal y, como menciona Trotta (2002), "la reestructuración del Estado está más vinculada a presiones exógenas que a decisiones endógenas, más cercana a condicionamientos para la recepción de futuros créditos (...) que a un proyecto de desarrollo autónomo".

Así, el Estado pierde soberanía y se vincula a una red de instituciones que llevan a cabo una especie de "governanza global" (García Delgado, 2003). La situación periférica de la Argentina en dicha red hace que ésta también pase, a su modo, por procesos de isomorfismo, en los que sigue las pautas presentadas por los países centrales y los organismos internacionales de créditos, delineando un isomorfismo típicamente coercitivo.

O tro rasgo del campo organizacional de las O NG s en Argentina es la baja profesionalización. Si bien a menudo siguen modelos administrativos de las grandes empresas privadas y de manuales de administración como los de Peter Drucker, la búsqueda de eficiencia-eficacia se ve impedida por la falta de crédito, lo que dificulta el mantenimiento de un permanente personal profesionalizado. Según un estudio realizado en el 2001 por Suárez, Pecci y Christensen, para cada empleado rentado que trabaja en una O NG hay 7.5 que trabajan voluntariamente. Las implicancias de ello están en que, primeramente, rara vez los voluntarios son elegidos en base a su formación profesional (Suárez, 1989), lo que hace que se de una gran rotatividad en las organizaciones. Ello, según los autores mencionados, remite a una institucionalización precaria, debido a la imposibilidad de dar una continuidad a los programas o de mirar la organización en función de un proceso a largo plazo (Suárez, et. al., 2001). 
Asimismo, un dato interesante que dicha investigación presenta es que incluso los directivos y ejecutivos de las organizaciones de la sociedad civil entrevistados tenían un bajo nivel educativo. De hecho, el 37\% de ellos sólo contaba con el nivel primario completo. Es importante notar que a pesar del surgimiento de varios cursos de posgrado y maestrías en el área de dirección de organizaciones del sector, fund raising, etc., la baja profesionalización señalada hace más de una década por Suárez no tuvo un cambio importante, al menos hasta el 2001, cuando el estudio fue realizado.

Los medios de comunicación también son un actor importante cuando se analiza el entorno de las $\mathrm{ONGs}$, ya que enfatizan temas que pueden tener éxito mediático, y los vincula a ciertas ONGs, como Cáritas, Red Solidaria, Greenpeace, entre otras, que logran llamar la atención hacia sí. También los organismos multilaterales determinan cuáles son los programas que pueden llegar a recibir crédito o financiación, cuál es la población objetivo que más lo necesita en el momento, cuáles serán las metodologías utilizadas para formular los proyectos, para ejecutarlos y monitorearlos.

Es decir, podemos considerar cuatro grandes actores que interactuaron con las O N Gs en Argentina, principalmente en la década del 90: el Estado, los organismos multilaterales de crédito y agencias internacionales en general, los medios de comunicación y las organizaciones privadas con sus modelos de eficacia-eficiencia. Es así como se vuelve esencial caracterizar el campo organizacional de las ONGs en la Argentina a fines de mostrar la complejidad del mismo y captar en su complejidad los procesos de institucionalización de las mismas. Al hablar de campo organizacional no "sólo se pretende identificar un grupo de organizaciones que crean productos 0 servicios similares, sino también se incluyen a los socio determinantes para sus intercambios, las fuentes de financiamiento, los grupos reguladores, las asociaciones profesionales y otras fuentes de influencia" (Scott, 1991). 


\section{El isomorfismo periférico}

El primer intento de abordaje a la bibliografía acerca de las O N Gs en Argentina fue en función de las categorías de isomorfismo propuestas por Powell y Di Maggio, para ver como los datos se adecuaban a ellas, es decir, ver en qué medida se podía identificar isomorfismos coercitivos, miméticos y normativos. Vimos, entonces que el campo organizacional de las O NGs en Argentina contaba con ciertas especificidades que hacían que nuestras herramientas teóricas fueran insuficientes o inadecuadas y, a menudo, hacía falta forzar el dato en la categoría. Luego, a partir de un análisis de dichas especificidades, llegamos a nuevas categorías formadas a partir de los datos. Primero, una categoría bastante general que da cuenta de la gran mayoría de las O N Gs de Argentina a la denominaremos isomorfismo periférico.

Con eso nos referimosa la necesidad de adaptarse a los requerimientos e imposiciones, directas o indirectas, de los países centrales, a través de las determinaciones de organismos multilaterales los que se pide financiación. La Relatoría del Encuentro "Entre el asistencialismo y el desarrollo: El rol de las ONG 's en el momento actual de América Latina y Argentina"(1989), por ejemplo, subrayaba la importancia de la vinculación entre O N Gs y Estado en los países en vías de desarrollo, aunque en la mayoría de las veces no es el Estado el que aporta financiación a aquéllas. Mencionaba también que este vínculo, así como la capacidad de replicabilidad de los proyectos, son fundamentales para el trabajo de las $\mathrm{ONGs}$, aunque su independencia de criterio y de acción se vea limitada o que no puedan desarrollar mecanismos de presión que impacten en el Estado. Todo eso, según dicha relatoria, hace que las O NGscuenten con escasa autonomía para definir sus propias agendas.

Refiriéndose específicamente acerca de las dificultades de financiación, en "El Perfil de las Ong's en la Argentina", Filmus, Arroyo y Estebanez (1997) mencionan la capacidad que las organizaciones más grandes tienen para captar recursos desde afuera, generando, así, una fuerte competencia 
por la captación de recursosque son, a su vez, escasos. Estos autores señalan, asimismo, que a menudo son los organismos internacionales quienes proveen los criterios de evaluación y de control de gestión de los fondos otorgados por ellos, de modo que las organizaciones pequeñas o de base sufren exigencias demasiado exageradas para su propia capacidad y terminan flexibilizándose y desarticulándose de las políticas públicas concretas.

La dependencia de organismos extranjeros también puede ser vista en las definiciones de las $\mathrm{O}$ N $\mathrm{s}$ o de programas de investigación acerca de las mismas, ya que muchos de estos son llevados a cabo por financiación y/o iniciativa extranjeras. En "Estudios sobre el Tercer Sector sin fines de lucro en Argentina", Roitter (2000) hace una descripción de la metodología utilizada por un proyecto comparativo del sector sin fines de lucro que, luego de estudiar países como EE.UU., Reino U nido, Francia, Alemania, Italia, Suecia, Hungría y Japón, aplica la misma definición y sistema de clasificación para países de América Latina, elaboradosen colaboración con asociados locales.

Esta adaptación a definiciones de organismos internacionales también puede ser encontrada en el trabajo del PNU D-BID "El Capital Social. Hacia una construcción del índice de desarrollo de la sociedad civil en Argentina" (1998), en el cual definen los indicadores utilizados para medir dicho desarrollo, como antigüedad del sector; capacidad de respuesta frente a cambios en los beneficiarios; liderazgos; sus necesidades; los procesos de descentralización; gestión de las políticas sociales, entre otros. Esta búsqueda de la cuantificación del desarrollo del tercer sector en Argentina, debido a las propias vicisitudes del método, si bien gana posibilidades de comparación, pierde en lo que se refiere a una descripción más precisa de las O NGs. Las organizaciones, a su vez, adoptan esos mismos indicadorescomo estándares de su propio desempeño, principalmente si consideramos la importancia de dichos organismos para la otorgamiento de crédito y de definición de lineamientos. 
Sociologias, Porto Alegre, ano 8, no 16, jul/dez 2006, p. 216-249

Las implicancias de esa dependencia tanto de financiación externa, como del Estado (a nivel nacional, regional o local), hace que las ONGs, para su propia supervivencia, adécuense a las definiciones y exigencias impuestas desde afuera. Frente a la situación de escasez de recursos y de delegación de responsabilidades al tercer sector, por parte del Estado, de un modo y otro, las O NGs terminan perdiendo autonomía y motivación a la creatividad.

Todas las ONGs, de un modo u otro, comparten tales presiones isomórficas, que es una categoría que se refiere al contexto macro económico y social donde se insertan las organizaciones. En este sentido, la opción por seguir patrones isomórficos resulta de las dificultades con las que las O NGs tienen que convivir, propias de una sociedad periférica que, no obstante, participa activamente de los procesos de globalización, incrementándose, de este modo, dicha complejidad.

Luego, en el seno del isomorfismo periférico, podemos discernir dos tipos o categorías más específicos: el isomorfismo coercitivo y el isomorfismo mimético.

\section{El isomorfismo coercitivo}

Cuando Powell y Di M aggio hablan de isomorfismo coercitivo se refieren más bien a la imposición directa por parte del Estado o de otra organización para que se produzca un cambio: "en algunas circunstancias, el cambio organizacional es la respuesta directa a un mandato del gobierno: los fabricantes ad optan nuevas tecnologías para controlar la contaminación a fin de sujetarse a las regulaciones gubernamentales. Pfeffer y Slancik (... ) observan que los ambientes construidos políticamente tienen dos características: a menudo los que toman las decisiones políticas no experimentan las consecuencias de sus acciones; y las decisiones políticas se aplican de manera 
general a toda clase de organizaciones, lo que hace esas decisiones menos adaptativas y menos flexibles". En el caso de las O N G sargentinas, el Estado no cumple un rol controlador acerca de qué hacen las ONGs y cómo lo hacen (aunque la situación de las organizaciones privadas pueda ser distinta). Además, el ambiente institucional de las O N Gs no cuenta con actores tan fuertes que puedan imponer modelos a través de la coerción. Así el isomorfismo coercitivo adquiere características diferentes a las mencionadas por lo autores, pero, principalmente, más específicas. Acá el isomorfismo coercitivo se refiere a imposiciones o presiones que las O NGs sufren de actores externos a las que se encuentran frente a la necesidad de realizar cambios organizacionales para alcanzar la legitimación.

En ese sentido, se podría hablar de dos tipos, aún más específicos, de isomorfismo coercitivo: isomorfismo político o clientelístico e isomorfismo jurídico. El primero se refiere a los cambios que las ONGs tienen que hacer para adaptarse a los gobernantes de turno así como a las políticas sociales, muchas veces valiéndose de prácticas clientelistas, o sea, las O NGs deciden formar parte de las estructuras clientelares de otras ONGs, de algún partido político o de algún funcionario público en especial, ya que con ello se ven, de algún modo, favorecidas. Ello también se debe a que a veces se crean o se deshacen ministerios o secretarías. Además, una nueva política social desarrollada por el Estado puede necesitar el apoyo de diferentes 0 N Gs y éstas necesitan estructurarse de una manera diferente para adecuarse a la demanda. Esto puede ser observado más bien en las prácticas específicas de las organizaciones, también en un nivel más local, diferentemente del isomorfismo periférico pero, sin duda, como parte de éste.

Thompson (1995), en "Público y Privado. Lasorganizaciones sin fines de lucro en la Argentina", a su vez, aclara que esta vinculación entre Estado y sector privado o social como proveedores de crédito propicia el surgimiento de relaciones clientelistas, cooptativas y de competencia entre las organizaciones más de base. 
Sociologias, Porto Alegre, ano 8, no 16, jul/dez 2006, p. 216-249

Trotta (2002) define el clientelismo como una relación social basada en la desigualdad; es una relación cara a cara, y que tiene una flexibilidad difusa, es decir, que no se encuentran claramente definidos los términos de la relación. Él dice que la reciprocidad es la principal característica de este tipo de práctica, ya que es aquélla la que mantiene el vínculo entre el patrón y el cliente. Estas serían características fijas del clientelismo. Por otro lado, las características variables del clientelismo, que van a configurar su particularidad, son: "el origen de la iniciativa para establecer la relación, la duración en el tiempo de la misma, el alcance de la relación y su intensidad".

Este autor hace una diferenciación entre "clientelismo institucional" o "clientelismo fino", en oposición al "clientelismo tradicional" o "duro". En el primero, el recurso que se utiliza en la relación es implícito y no tangible, como puede ser incluir determinadas personas en un determinado plan social, y eso hace, por otro lado, que la relación sea más larga que en el clientelismo tradicional, ya que el cliente se ve involucrado en una red de estructuras de decisiones que hacen que el alcance territorial del clientelismo fino sea más amplio que el tradicional. A su vez, el clientelismo tradicional tiene objetos tangibles (muchas veces comida, ropa, etc.) como recursos en la relación. Ésta es más bien corta y tiene un alcance más que nada barrial. Cuando hablamos, entonces, del clientelismo como parte del isomorfismo coercitivo, nos referimos esencialmente al clientelismo institucional.

Así, el trabajo de Martori (1996), "El servicio de las organizaciones comunitarias", ofrece un claro ejemplo de los antedicho cuando describe la contradicción existente entre las prácticas de implementación de políticas sociales por parte del Estado y aquellas de las organizaciones comunitarias surgidas del trabajo en el barrio. Eso llevó a que el trabajo social quedara en manos de los punteros políticos que, mientras aseguran el funcionamiento del sistema, controlan las demandas y oferta de bienes y servicios sociales y refrenan el trabajo de las organizaciones locales. 
Debe señalarse que el isomorfismo político tiene características particulares en relación con los otros tipos de isomorfismo, en tanto los efectos que tiene en la estructura organizacional. Es decir aquellas $0 \mathrm{NG}$ G en donde se observan presiones isomórficas de tipo político, se da una estructura cuyos criterios difieren de los de aquellas en donde las presiones apuntan a una estructura similar a la empresa privada, en tanto eficiencia y funcionamiento de la misma; no se apunta a un tipo de racionalidad de tipo racional legal sino que intervienen factores ligados a las relaciones de tipo clientelares. Las organizaciones adquirirían ciertas características estructurales no por decisión o diseño racional, sino por considerarse una manera de lograr la supervivencia de la organización, a través de prácticas extendidas en la región.

Asimismo, es importante tener en cuenta la baja institucionalidad existente en los aparatos gubernamentales y el espacio que existe para la discrecionalidad. Es decir, la existencia de discrecionalidad y patrones de relaciones personalizadas dan lugar al clientelismo.

A ese respecto, Mónica Palacio, en "El caso de una organización de desocupados en la provincia de Rio Negro" (1997), mencionaba como las organizaciones de los sectores marginales dependían intensamente de las políticas sociales implementadas por el Estado y que, en última instancia, es el Municipio el que termina garantizando las posibilidades de acceso al empleo y a la subsistencia. Eso muestra tal dependencia de la organización frente al Municipio que lo que ocurre a éste, indefectiblemente, impactará en el desarrollo de aquélla.

A su vez, dentro del isomorfismo coercitivo encontramos el isomorfismo jurídico, que se refiere a la necesidad de adaptarse a leyes vigentes para que la ONG sea considerada como tal, dejando de lado la informalidad (lo que, consecuentemente, debe facilitar el acceso a financiación). Entre los procedimientos de inscripción se incluye, a menudo, 
Sociologias, Porto Alegre, ano 8, № 16, jul/dez 2006, p. 216-249

el pago de aranceles, la necesidad de tener personería jurídica, con estatutos, demostración patrimonial (para el caso de las asociaciones civiles y fundaciones) e inscripción en determinados organismos públicos (estatales, provincialeso municipales). Así, las organizaciones, en la misma elaboración de su autodefinición, se encuentran frente a la necesidad de adaptarse a la estructura jurídica existente. Es decir, la misma organización tiene que encargarse de tener ciertas características o propiedades para que pueda "llenar los casilleros" de los formularios que necesita en la Dirección Provincial de Personas Jurídicas, por ejemplo, o de una organización a la que va a solicitar financiación.

Diferentes trabajos mencionan este tipo de necesidad. Bellardi y De Paula (1986), en "Villas M iseria: origen, erradicación y respuestas populares", subrayaban como las cooperativas instaladas en las villas miseria estudiadas se otorgaron una organización legal-administrativa, tal como un estatuto y una inscripción en el Instituto Nacional de Acción Cooperativa, para asegurar la continuidad y seriedad del sus proyectos. Dígase de paso, lo que las autoras denominan "seriedad", podemos llamar de legitimación $y$, vemos, como el isomorfismo, en este caso jurídico, funciona como un mecanismo que les garantiza la misma.

En un texto oficial del CENO C, "H acia la constitución del tercer sector en Argentina" (1997), también se aclara que las ONGs que no estén registradas en el Registro N acional de Entidades del Bien Público, que otorga personería jurídica a las $\mathrm{O}$ NGs, no pueden acceder a la recepción de subsidios por parte del gobierno federal. Los trabajos de Regnner y Silua (1994) así como de Filmus, Arroyo y Estebanez (1997) también hacen menciones a este mismo punto.

En lo que se refiere al isomorfismo jurídico, es importante mencionar que la misma definición de la organización (como mutual, asociación, fundación, sindicato, etc.) se ve dificultada por el hecho de que la legislación 
no ha acompañado el fuerte desarrollo que se dio en el sector en la década del noventa. Así, las definiciones de O NGs, organizaciones sin fines de lucro, asociaciones, etc.. suelen ser ambiguas o demasiado abarcadoras. A esto se refiere, específicamente, Roitter (2000), en su trabajo ya mencionado, cuando dice que "la falta de una ley de asociaciones civiles - que es una de las formas legales principales que asumen las organizaciones sin fines de lucro en la Argentina - sumado al hecho de que las normas del Código Civil son demasiado generales y se revelan insuficientes ante la complejidad del campo genera verdaderos vacíos legales que promueven la generación de espacios de discrecionalidad".

Como vemos, entonces, el isomorfismo coercitivo se refiere, para el caso puntual de la Argentina, a dos principales formas de adaptación que las O N Gs tienen que sufrir, hasta cierto punto, inevitablemente: al entramado político en el cual se inserta (municipal o barrial) y a la legislación vigente. Así, el cambio organizacional que ello conlleva, en términos jurídicos y de gestión, garantiza la perpetuación del trabajo desarrollado por las 0 NGs, es decir, asegura su legitimación.

Vale reforzar que las O NGs pueden optar por no participar de esos entramados político-clientelares o por no inscribirse en el Registro $\mathrm{N}$ acional de Entidades de Bien Público, de modo que no hay una coerción real hacia esas prácticas. Pero en estos casos, la no participación implica, de forma más perentoria, un perjuicio a la actividad de la organización.

\section{El druckerianismo endémico y las modas temáticas}

En lo que concierne al isomorfismo mimético, para Powell y Di M aggio constituye una "una respuesta a la incertidumbre" y dan ejemplos de innovaciones que las empresas hacen para reforzar su legitimidad, "para demostrar que por lo menos tratan de mejorar las condiciones de trabajo". 
Sociologias, Porto Alegre, ano 8, no 16, jul/dez 2006, p. 216-249

Además, dicen que "las organizaciones tienden a modelarse a sí mismas siguiendo organizaciones similares en su campo, las cuales les parecen más legítimas o exitosas".

En este caso, para Argentina se dan dos situaciones. Primero, un isomorfismo gerencial (druckeriano), en el que se siguen mitos organizaciones propugnados por escuelas de management estadounidenses para gerenciar una ONG, sea basándose en los éxitos de organizaciones lucrativas o siguiendo manuales de administración de empresas, con especializaciones en fund raising, etc. Hay que tener en cuenta que el manual de Peter Drucker (1990) acerca de la dirección de non-profits se basa fundamentalmente en ejemplos de organizaciones estadounidenses, a menudo firmemente establecidas y de gran porte, como hospitales, universidades de gran porte (como Stanford) y fundaciones como Kellogg o Rockefeller, que difieren mucho de las O NGs argentinas no sólo en términos de tamaño y en su forma de conseguir financiación, pero principalmente en cómo están ancladas en el entretejido social. La historia de las nonprofits tiene que ver con la misma historia de los Estados U nidos, ${ }^{4}$ de su cultura, etc., de la misma manera que las ONGs tienen que ver con el desarrollo histórico de Argentina, como ya se mencionó anteriormente.

Algunos de los preceptos descriptos por D rucker son: la necesidad de centrarse en la misión de la organización, fijando metas concretas para la acción; la importancia que debe ser dada al liderazgo adentro de la organización para lograr resultados, la gerencia debe volcarse hacia el rendimiento; el papel fundamental de la junta directiva, de los voluntarios,

\footnotetext{
4 En lo que se refiere a los Estados U nidos, mucho se ha escrito desde Alexis de Tocqueville acerca de la amplia participación, incluso desde el período colonial, en organizaciones, ligas, asociaciones voluntarias, etc., lo que daría un tinte participativo a la democracia estadounidense. O rganizaciones por los derechos de los afro-americanos, de las mujeres, por causas ecológicas, entre otras, surgieron en los Estados Unidos en épocas bastante tempranas, y si bien hoy diversos estudios muestran una participación decreciente en este tipo de asociaciones, ellas han otorgado rasgos muy particulares a la sociedad civil y su forma de organizarse en aquel país (Skocpol y Fiorina, 1999).
} 
etc. En nuestra bibliografía, encontramos inumerosos ejemplos a ese respecto.

En el "M emoria del IV Encuentro Iberoamericano del Tercer Sector" (1998) se señala la capacidad que las O NGs deben tener para responder rápidamente a los cambios, cumpliendo, antes que nada su misión. Aclara también que la diferencia entre el sector público, privado y el tercer secot es cada vez más difusa y unos de los motivos es que las organizaciones del tercer sector tienden a incorporar técnicas del mundo empresarial para mejorar su eficacia y el impacto de sus actividades, por un lado, y, por otro, dada la búsqueda de auto-sustentabilidad, optan por prestar servicios que terminan asemejándolas a empresas comerciales.

Cahián, en "Derecho de las fundaciones" (1996), habla de la necesidad que las 0 ngs tienen de utilizar la tecnología moderna para mejorar la calidad y la gestión institucional. A algo parecido se refiere Chopourian, en "Alianzas entre empresas y el Tercer Sector" (1998), que dice que las O NGs sólo pueden ser más eficientes mediante alianzas estratégicas con el sector privado y las herramientas del management deben ser aplicadas a la gestión de aquéllas.

En cierto punto, esto se funde con lo que Powell y DiM aggio dijeron acerca del isomorfismo normativo. El isomorfismo normativo se refiere principalmente a la profesionalización, que definen como "la lucha colectiva de los miembros de una ocupación por definir las condiciones y métodos de su trabajo (...) y por establecer una base cognitiva y la legitimidad de su autonomía ocupacional"; "un mecanismo relevante para alentar el isomorfismo normativo es la filtración de personal", "la profesionalización de la gerencia tiende a ocurrir junto con la estructuración de los campos organizacionales".

El desarrollo del campo de las O N Gs en Argentina no ha alcanzado todavía tal nivel de profesionalización. En verdad, como ya se mencionó, una deficiencia de las $\mathrm{O}$ N Gs de los países subdesarrollados es justamente la dificultad para manejar los recursos humanos, debido a la falta de 
Sociologias, Porto Alegre, ano 8, no 16, jul/dez 2006, p. 216-249

especialización que tienen y también al financiamiento discontinuo, que hace que no se pueda mantener empleados asalariados y se use tantos voluntarios no profesionales (Suárez, 1989). Por lo tanto, el isomorfismo normativo, tal como explican los autores, es un fenómeno poco difundido.

Sin embargo, los modelos de gerencia propuestos por manuales (tipo el de Drucker) y por gurues empresariales muchas veces son considerados como metas para alcanzar una determinada eficiencia.

Varios de los materiales estudiados aquí mencionan la necesidad de formación o capacitación para mejorar la eficiencia de las O NGS. El trabajo de Federico Cerdeiro, "Aprendizaje en organizaciones sin fines de lucro"(1999), habla de la importancia de la capacitación en las O N Gs para que ellos puedan administrarse con estándares semejantes a las organizaciones lucrativas para lograr los avances que éstas han logrado.

La publicación de la Fundación Grupo Sophia, "O rganizaciones de la Sociedad Civil. Desafíos de gestión de desarrollo de capital social" (1998), además de hacer un fuerte hincapié en las herramientas gerenciales "druckerianas", enfatiza la necesidad de capacitación para fortalecer la capacidad de acción de las organizaciones. También Cáritas San Isidro, en su exposición de la Jornada Argentina del Sector Social - "Alianzas: la unión hace la diferencia" (1997) - mencionaba que el crecimiento de la organización vino a partir de la necesidad de organizarse y capacitarse, y que la capacitación es fundamental para trabajar en los sectores excluidos.

O tro tipo de isomorfismo que se incluiría en el mimético sería el isomorfismo temático, que se refiere al hecho que las O NGs se dediquen a temas de interés mediático 0 de interés de los organismos multilaterales de créditos, lo que hace que muchas, adentro de la heterogeneidad característica del sector, trabajen sobre un tema determinado al mismo tiempo, es decir, se mimeticen en función del tema para garantizar la supervivencia de la organización. Además de ello, como las O NG s pueden mimetizarse en función de los requerimientos que los organismos internacionales y/o 
multilaterales de crédito exigen en el momento en que deben llenar los formularios para pedir financiación, no sólo terminan por llevar a cabo actividades que reconocidamente consiguen el crédito, sino que en definitiva se adecuan a las definiciones de dichos organismos.

El tema puede surgir a partir de un hecho histórico determinado, como fue el caso de las ONGs que trabajaban en la defensa de los derechos humanos luego del fin de la dictadura en Argentina, o como puede ser el surgimiento de muchas $\mathrm{ONGs}$ dedicadas a conseguir bienes de primera necesidad luego de un desastre natural, como inundaciones o terremotos. Asimismo, pueden surgir de "modas" originadas en países centrales que son difundidas en los países periféricos, como la responsabilidad social o de la defensa del medio ambiente.

El trabajo de Filmus, Arroyo y Estebanez (1997) antedicho, se refiere a "Ias cambiantes condiciones del financiamiento obligan a las O NGs a un importante margen de flexibilidad para responder a las cambiantes 'modas' de financiamiento de los organismos internacionales" Afirman, ademásque eso hace que las organizaciones queden muy vulnerables y dependientes de la construcción 'externa' de agendas en vez de centrarse en proyectos que busquen solucionar problemas de la comunidad.

En lo que se refiere a los temas, pudimos ver que las O N Gs en los 90 trabajaban más que nada en el ámbito local, siguiendo a la tendencia a la búsqueda del desarrollo local que empezó a prevaler en esa época (García Delgado, 2003). Ello puede ser ejemplificado con la Relatoría del Encuentro "Entre el asistencialismo y el desarrollo: El rol de las ONG 's en el momento actual de América Latina y Argentina " (1989), donde se afirma que las ONGs se centran más que nada en lo local y en lo barrial y a menudo carecen de una visión de conjunto. También se subraya que las ONGs tendría que tener un mayor peso para definir sus propias agendas en función de los problemas básicos de su país.

En la tesis "visibilidad social, medios de comunicación y O NGs: un estudio de caso", de Alan Courtis (2000), se habla de la capacidad que las 
Sociologias, Porto Alegre, ano 8, no 16, jul/dez 2006, p. 216-249

O NGs tienen de instalar en la opinión pública temas de interés general como forma de ejercer presión en los mecanismos decisorios. Este es un ejemplo del rol de los medios de comunicación como sensibilizadores de la sociedad repecto a las problemáticas abordadas por las organizaciones. A este respecto el trabajo de Thompson, "Público y Privado. Las organizaciones sin fines de lucro en la Argentina" (1995), también afirma que "los medios de comunicación social juegan un rol preponderante en la estrategia de sensibilización, información y denuncia de esas organizaciones. Con temas 'seductores' para un amplio sector de la sociedad, utilizan tecnologías más innovadoras (de marketing, segmentación de público) que renueva el modelo de comunicación con la gente".

Vemos así que el isomorfismo mimético (gerencial o temático) también adquiere rasgos peculiares al entorno específico que estudiamos. Lo principal acá es que los desafíos impuestos por el entorno, principalmente por la incertidumbre característica del sector en Argentina, en lo que se refiere a recursos humanos y materiales, eficiencia, eficacia y visibilidad. La opción por el mimetismo, para asegurar la legitimidad, tiene en cuenta todos esos retos y busca, de esta forma, dar respuesta a ellos.

Las categorías planteadas de isomorfismo periférico pueden ser graficadas como sigue:

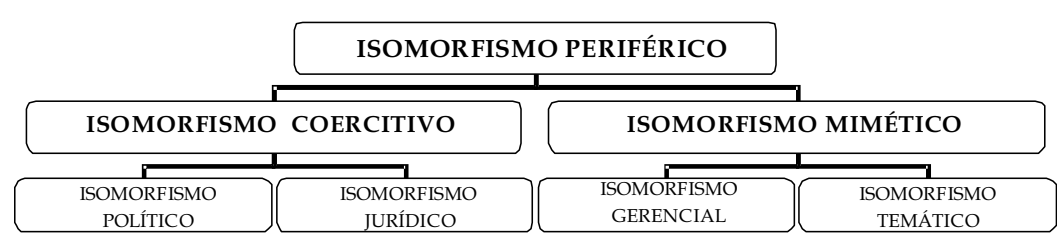


Sociologias, Porto Alegre, ano 8, no 16, jul/dez 2006, p. 216-249

\section{Conclusiones}

Considerando la importancia que la teoría neoinstitucional le otorga la relación entre la organización y el entorno para la institucionalización, optamos por observar el entorno de las O N Gs en Argentina ya que este debería ser definitivamente diverso a aquél de las organizaciones tratadas por los autores de otros países. Sin duda, vimos que el entorno, con sus peculiaridades locales, imprime rasgos muy particulares a la institucionalización de las ONGs en nuestro país. Podríamos preguntarnos acerca de si esta descripción y nuevas categorías a las que llegamos son compartidas por los países en vías de desarrollo, ya que muchos, principalmente en América Latina, pasaron por procesos bastante similares en lo que se refiere a la reestructuración del aparato estatal y la descentralización de las políticas públicas.

En el proceso de institucionalización, las organizaciones en general, y las ONGs, considerando nuestro caso particular, se hallan frente a la necesidad de incorporar mecanismos que las legitimen. Uno de esos mecanismos es el isomorfismo que hace que se produzca una homogeneización del campo organizacional. Dado que las organizaciones se relacionan con su entorno de modo que éste influye en aquélla y vice-versa, las características de dicho entorno van a definir como se dan los procesos de isomorfismo.

El campo de las ONGs en Argentina, principalmente a partir de la crisis del Estado de bienestar, cuenta con una institucionalización débil, debido a la inconstancia en la financiación y en el mantenimiento de sus recursos humanos. Luego, la opción (¿o sumisión?) por una homogeneización (isomorfismo), donde se busca cumplir con pautas de la eficacia-eficiencia, con las leyes que determinan su status jurídico, etc., al menos hacia afuera, la incorporación de mitos organizacionales racionalizados les otorga cierta legitimidad frente a la sociedad y a los organismos otorgadores de crédito. 
Sociologias, Porto Alegre, ano 8, no 16, jul/dez 2006, p. 216-249

Sin embargo, si la legitimidad se da en detrimento de la eficiencia, es importante cuestionarse hasta qué punto esa legitimidad es válida como para que las 0 NGs vengan a cumplir las funciones que antes tenía el Estado. ¿Hasta que punto logran ser más eficientes que el Estado?, principalmente a medida que ambos se encuentran en una situación de dependencia externa. ¿El isomorfismo, al final, no termina por hacer que las O NG s hagan lo mismo que hizo el Estado cuando, en pos de una supuesta eficaciaeficiencia, delegó las políticas sociales a otras instancias?

De cualquier modo, si bien Berger y Luckmann (1997.) dicen que la legitimación no es un paso obligatorio de la primera fase de la institucionalización, sí lo es cuando las objetivaciones tienen que ser transmitidas a nuevas generaciones. Así, para que las O NGs perduren en el tiempo en tanto instituciones legítimas, la búsqueda de legitimación a través de mecanismos isomórficos es fundamental.

O tra pregunta que nossurgió es acerca de los procesos de legitimación por el cual incurren las organizaciones de la Iglesia Católica, como Cáritas, que aparentemente no ubicaría en ningún de los cuatro tipos de isomorfismos específicos que mencionamos, ya que siguen una trayectoria paralela a las O NGs en general.

Es importante aclarar que sería ingenuo creer que los procesos de isomorfismo, sean miméticos o coercitivos, se dan a través de una imposición externa que la O NG sufre sin alternativas. Aunque sea la única salida aparente, el isomorfismo es una estrategia que la ONG adopta, intencionalmente o no, para relacionarse con su entorno buscando, así, legitimarse. De modo que las O N Gs no son actores pasivos, sino que actúan para lograr sus objetivos, usando las herramientas que tienen al alcance de sus manos. "La sumisión a ciertos fines, significaciones o intereses trascendentes, es decir, superiores o exteriores a los intereses individuales, raramente es un efecto de una imposición imperativa y de una sumisión consciente. (... ) La 
subordinación del conjunto de las prácticas a una misma intención objetiva, especie de orquestación sin maestro, sólo se da a través de la concordancia que se instaura (... ) entre lo que estos son y lo que hacen (... ); concordancia que puede expresarse en el sentimiento de estar bien 'en su lugar', de hacer lo que se tiene que hacer (...) o en la convicción resignada de no poder hacer otra cosa, lo que también es una manera, sin duda menos feliz, de sentirse destinado a hacer lo que se hace" (Bourdieu, 2003).

Si bien las eleccionesya están previamente determinadas por la misma estructura del campo organizacional y las organizaciones, a su vez, cuentan desde el principio con diferentes recursos, siempre les queda un margen (aunque pequeño) de maniobra. Ello nos remite directamente al pensamiento neoinstitucional y su afirmación de que se da una dialéctica entre el individuo y su construcción social y la estructura.

\section{Referências}

ARROYO, D. "El cambio de la estructura social y las nuevas formas de organización en Argentina". Artículo para el curso "Sociedad Civil y Tercer Sector", desarrollado en FLACSO - Buenos Aires, en 2003. Sin año.

BELLARDI, M. y DE PAULA, A. Villas Miseria: origen, erradicación y respuestas populares. Buenos Aires: Centro Editor de América Latina. 1986.

BERGER, P. \& LU CKM AN N, T. La construcción social de la realidad. BuenosAires: Amorrortu. 1997.

BO URDIEU, P. “Le mort saisit le vif. As relações entre a historia reificada e a história incorporada". In: O Poder Simbólico (2003). Bertrand Brasil: Rio de Janeiro. 1980.

CAHIÁN , A. y PO RTO , A. Derecho de las fundaciones. Ediciones La Roca. Buenos Aires, 1996.

CEN O C. Hacia la constitución del tercer sector en Argentina. Las actividades de las organizaciones de la comunidad inscriptas en el CENOC 1997. Buenos Aires: Presidencia de la Nación, Secretaría de Desarrollo Social. 1997. 
Sociologias, Porto Alegre, ano 8, ํo 16, jul/dez 2006, p. 216-249

CERDEIRO, F. Aprendizaje en organizaciones sin fines de lucro. Trabajo de licenciatura - Universidad San Andrés. 1999.

CHO POU RIAN, F. "Alianzas entre empresas y el tercer sector: un nuevo modelo de responsabilidad corporativa". Trabajo de Licenciatura, Administración de Empresas, U niversidad de San Andrés, Buenos Aires. 1998.

COASE, Ronald H. "The New Institutional Economics". Journal of Institutional and Theoretical Economics 140:229 - 231. 1983

DE PIERO, S. "Sociedad Civil y Tercer Sector en Argentina: definiciones y debates teóricos". Artículo para el curso "Sociedad Civil y Tercer Sector", desarrollado en FLACSO - Buenos Aires, en 2003. Sin año.

"El perfil del Tercer Sector en la Argentina". Artículo para el curso "Sociedad Civil y Tercer Sector", desarrollado en FLACSO - Buenos Aires, en 2003.

DIM AGGIO, Paul \& PO WELL, Walter, W. "The Iron Cage Revisited: Institutional Isomorphism and Collective Rationality in O rganizational Fields". American Sociological Review 48:147-160.1983

"Introduction." Pp. 1-38 In: The New Institutionalism in Organizational Analysis, edited by PO W ELL, Walter W. and DIM AGGIO, Paul J. Chicago: University of Chicago Press. 1991.

DRU CKER, P. Dirección de instituciones sin fines de lucro. Editorial Ateneo: Buenos Aires.

Encuentro iberoamericano para el Tercer Sector. Hacia un nuevo contrato social para el siglo XXI, 1998. Memoria - actas del encuentro. Buenos Aires. 1990.

FILMUS, D. (Coord.); ARRO YO , D.; ESTEBANEZ, M. E. El Perfil de las ONG's en la Argentina. FLACSO-Banco Mundial. 1997.

FO RNI, P. "Las redes inter-organizacionales y el desarrollo de las O NGs de base. Estudios de caso en el Gran Buenos Aires durante la década del 90". In: Organizações \& Sociedade, $n^{\circ}$ 20, U niversidade Federal da Bahía, Brasil. 2001.

"The Creation of Poverty NGOs in Latin America. A Case Study in Greater Buenos Aires". Tesis de doctorado. University of Notre Dame, Estados Unidos. 2000. 
FU NDACIÓ N GRUPO SO PHIA. O rganizaciones de la Sociedad Civil. Desafíos de gestión y desarrollo del capital social. Buenos Aires: Fundación Grupo Sophia. 1998.

GARCÍA Delgado, D. Estado-nación y la crisis del modelo. El estrecho sendero. Grupo Normal Editorial, Buenos Aires. 2003.

GIDDENS, Anthony. La construcción social de la realidad: bases para la teoría de la estructuración. Buenos Aires: Amorrortu. 1984.

GÓ MEZ del Río, M. (comp.). La unión hace la diferencia. Tercera Jornada Argentina del Sector Social. Buenos Aires: Fundación Compromiso. 1998.

JEPPERSO N, Ronald L. "Institutions, Institutional Effects, and Institutionalization". Pp. 143-163 In: The New Institutionalism in O rganizational Analysis, edited by PO WELL Walter W. and Paul J. DiMaggio. Chicago: University of Chicago Press. 1991.

MARTO RI, A. El servicio social en las organizaciones comunitarias. Trabajo de investigación final, Escuela Diocesana de Servicio Social, U niversidad de Morón, Buenos Aires. 1996.

MEYER, John W. and SCOTT, Richard W. "Centralization and the Legitimacy Problems of Local Government". Pp. 199-215 In: Organizational Environments: Ritual and Rationality, edited by MEYER, John W. and SCOTT, Richard W. Bervely Hills, CA: Sage. 1983.

MURTAGH, Ricardo (org.). Pobreza urbana en la Argentina. Editorial de la Universidad Católica Argentina, Buenos Aires. 2002.

PALACIO, M. El caso de una organización de desocupados en la provincia de Rio Negro. Tesis de Licenciatura, Escuela de Sociología, Universidad del Salvador, Buenos Aires. 1997.

PARSO NS, Talcott. A Sociological Approach to the Theory of Organization. Pp. 16-58 In: Structure and Process in Modern Societies, by Talcott Parsons. Glencoe, IL: Free Press. 1960a.

Some Ingredients of a General Theory of Formal O rganization, Pp. 59-96 In: Structure and Process in Modern Societies, by Talcott Parsons. Glencoe, IL: Free Press. 1960b. 
Sociologias, Porto Alegre, ano 8, no 16, jul/dez 2006, p. 216-249

PARSO NS, Talcott. Prolegomena to a Theory of Social Institutions. American Sociological Review 26:854-866. 1990.

PNUD - BID. El capital social. Hacia la construcción del índice de desarrollo de la Sociedad Civil en Argentina. Buenos Aires: Edilab. 1998.

REGNER, F. y SILUA, L. Caritas barriales: una visión alternativa como campo de trabajo social. Trabajo de investigación final, Escuela Diocesana de Servicio Social, Universidad de Morón, Buenos Aires. 1994.

REILLY, Charles A. (ed). New Paths to Democratic Development in Latin America: The Rise of NGO -Municipal Collaboration. Boulder, Colorado: Lynne Rienner Publishers. 1996.

RELATO RÍA DEL EN CUENTRO, Entre el asistencialismo y el desarrollo: El rol de las ONG's en el momento actual de América Latina y Argentina. 1989. Revista Medio Ambiente y U rbanización, p. 91-99. Buenos Aires. 1989.

ROITTER, M ario. Estudio sobre el Tercer sector sin fines de lucro en Argentina. Argentina/Estados U nidos: CEDES - The Johns H opkins U niversity. 2000.

SALOMON, Lester y ANHEINER, Helmut K. The Emerging Nonprofit Sector, Manchester University Press, Great Britain. 1996.

SKO CPO L, T. y FIO RINA, M. Making sense of the Civic Engagement Debate. In: Civic Engagement in American Democracy. Washington DC, Brookings Institution Press. 1999.

SCOTT, Richard W. Institutions and O rganizations. California: Sage Publications. 1995.

SELZNICK, Philip. (1948). "Foundations of the Theory of O rganization". American Sociological Review, 13:25-35.

___. Leadership in Administration. New York: Harper \& Row. 1957.

SUÁREZ, F. Problemas sociales y problemas de programas sociales masivo, CIDESOEA, Buenos Aires. 1989.

SUÁREZ, F.; PECCI, M.C.; CHRISTENSEN, A. Informe final. Vulnerabilidad y Foratlecimiento de las Organizaciones de la Sociedad Civil. CEN OC, Buenos Aires. 2001. 
Sociologias, Porto Alegre, ano 8, № 16, jul/dez 2006, p. 216-249

THOM ,PSON, A. (comp.). Público y privado: las organizaciones sin fines de lucro en la Argentina. Buenos Aires: U NICEF Losada. 1995.

TROTTA, M . E. V. La metamorfosis del clientelismo político. Contribución para el análisis institucional. Espacio Editorial, Buenos Aires. 2002.

WILLIAMSON, O liver E. Comparative Economic Organization: the Analysis of Discrete Structural Alternatives. Administrative Science Q uarterly 36:269-296. 1991

ZU CKER, Lynne G. The role of Institutionalization in Cultural Persistence. American Sociological Review 42:726-743. 1977.

\section{Resumen}

En este artículo, buscamos brindar elementos para un marco analítico para abordar las organizaciones del tercer sector en los países periféricos, observando principalmente sus idiosincrasias. Para ello, realizamos un análisis de la literatura producida en la Argentina en los años 90 a la luz del enfoque neoinstitucional en los estudios organizacionales, mostrando la existencia de fuerzas isomórficas resultantes de los diferentes actores involucrados en el proceso de conformación del tercer sector. Éstas pueden ser englobadas en un isomorfismo periférico que moldea las estructuras, procedimientos, relaciones externas y temáticas abordadas por las organizaciones del tercer sector.

Palabras-claves: organizaciones del tercer sector; teoria neoinstitucional; isomorfismo; sociologia de lãs organizaciones; O NGs. 
ARTIGOS

ARTICLES

\section{Development and legitimation of Third-Sector organizations in Argentina: Towards defining a peripheral isomorphism}

\section{Pablo Forni \& Lucimeire Vergilio Leite}

In this article we intend to offer elements for an analytical framing to study third-sector organizations in peripheral countries, particularly by observing their idiosyncrasies. In order to do that, we have carried out a review of literature produced in Argentina in the 1990s in the light of isomorphic forces resulting from distinct actors involved in the process of constitution of the third sector. Those forces can be seen within a peripheral isomorphism that shapes structures, procedures, external relations, and themes approached by third-sector organizations.

Key words: Third-Sector O rganizations, Neo-Institutional Theory, Isomorphism, Sociology of O rganizations, NGOs. 